\title{
Biomechanical analysis of local and global strengthening of gluteus medius
}

\author{
Matej Daniel, ${ }^{1}$ Jana Hornová, ${ }^{1}$ Karel Doubrava, ${ }^{1}$ Michaela Tomanová ${ }^{2}$ \\ ${ }^{1}$ Department of Mechanics, Biomechanics and Mechatronics, Czech Technical University in Prague, Faculty of Mechanical Engineering, Prague, Czech Republic \\ ${ }^{2}$ Rehabilitation Institute Brandys nad Orlici, Brandys nad Orlici, Czech Republic
}

Received: October 2016 Accepted: February 2017

Muscle activity improvement is an important component of any physical therapy, sport rehabilitation, and injury prevention. An ideal strengthening focuses on the whole body, while there are many exercises destined to strengthen individual muscle group, individual muscle, or even an individual muscle segment. These therapies can be applied to strengthen weakened muscles or to improve performance of a muscle system in given physical activity.

Gluteus medius is an important stabilizer of the pelvis controlling frontal plane alignment of the pelvis during walking and other functional activities. ${ }^{[1]}$ Weakness or dysfunction of gluteus medius is associated with many injuries of the lower extremity and abnormalities in the walking and running, ${ }^{[2]}$ e.g. Trendelenburg gait, iliotibial band syndrome, patellofemoral pain syndrome or anterior cruciate ligament injuries. The effect of weak gluteus medius on hip mechanics is well-described in clinical studies, ${ }^{[2,3]}$ and also supported by mathematical models. ${ }^{[4]}$

However, less is known how the muscle synergy is affected, if the selected muscle group is strengthened. The aim of this study was to investigate to what extend strengthening of individual muscle or muscle group would affect muscles activation. Our research questions are as follows:

1. How does local strengthening of single gluteus medius influence activity in other abductor muscles?

2. How does strengthening of the whole group gluteus medius and minimus influence activity in other abductor muscles?

3. How the hip joint load is affected by muscle strengthening?
The muscle activation and joint loading was estimated using the OpenSim software version 3.1 (Simbios, Stanford University, CA, USA). OpenSim is opensource software which allows developing, analyzing, and visualizing three-dimensional muscle-actuated models of the musculoskeletal system. ${ }^{[5]}$ By creating dynamic simulations of the movement which combine anatomical models with the physics of the musculoskeletal system, muscle and joint forces that are difficult to study through experimentation can be analyzed. ${ }^{[6]}$

In the present study, a generic muscle model with 23-degree of freedom and 92 muscle-tendon actuators was adopted (Figure 1a). ${ }^{[7]}$ The muscles attached over a large area were divided into individual muscle groups. Simulation was performed for one representative stride, and muscle activation was computed using inverse dynamic optimization, where the sum of muscle activation squared was taken as the optimization function.

Each muscle was confined not to extend maximum isometric force. As the principal function of gluteus medius is abduction, the activation in hip abductors was observed. The muscle-tendon actuators evaluated were as follows (Figure 1b): gluteus maximus (1 segment, glut max), gluteus medius (3 segments, glut med 1-3), gluteus minimus (3 segments, glut min 1-3), piriformis (pir), sartorius (sar) and tensor fasciae latae (tfl). The maximum force during the walking cycle in normal gait was reported in the first segment of gluteus medius (glut med 1) and this muscle actuator was chosen for local strengthening.

The advantage of simulation is in possibility of testing various scenarios, e.g. muscle strengthening in the present study. Muscle strengthening was imposed

Corresponding author: Matej Daniel, PhD. Department of Mechanics, Biomechanics and Mechatronics, Czech Technical University in Prague, Faculty of Mechanical Engineering, 16000 Prague, Czech Republic. e-mail: matej.daniel@fs.cvut.cz 
(a)

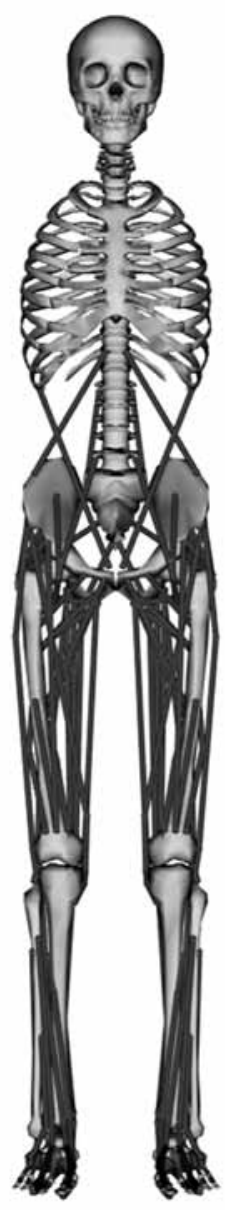

(b)

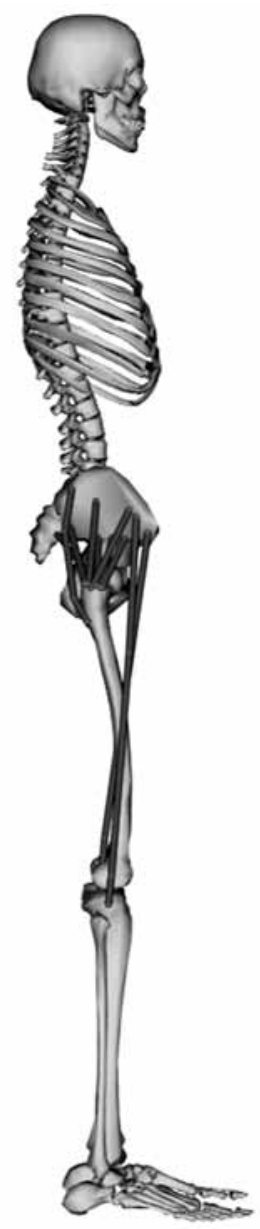

Figure 1. Musculoskeletal model. (a) Frontal view showing all muscles, (b) Lateral view showing examined muscles.

two ways. First, the maximum isometric force in single muscle-tendon actuator (glut med 1) was doubled to study the effect of localized strengthening. Second, all muscle actuators corresponding to principal muscle abductors gluteus medius and minimus ( 6 actuators) were strengthened by doubling their respective maximum isometric force. All muscle actuator parameters other than maximum isometric force were kept constant. Tendon stiffness and passive muscle stiffness are scaled to maximum muscle force in the OpenSim model ${ }^{[4]}$ and were, therefore, considered as increased in the strengthened muscles. The hip joint force was calculated considering the equilibrium of torques and forces acting in the hip joint. The input motion data and contact forces were taken from the OpenSim database (model gait 2392). A normal subject with a body weight of $72.6 \mathrm{~kg}$ and a height of $180 \mathrm{~cm}$ was considered.

Localized strengthening of one muscle actuator (glut med 1) overloads this muscle by $29 \%$, while it decreases activity of almost all other hip abductors (Figure 2). Global strengthening of the musculus medius and minimus renders the muscle activation to be more uniform by dividing the required force between the muscles (Figure 2). Hip joint force is almost insensitive to muscle strengthening (Figure 3).

In the present study, muscle-actuated dynamic simulations were used to identify how individual muscle strength influenced the muscle forces distribution during gait. Musculoskeletal simulation was adopted, as it provides controlled environment, elucidating cause and effect relationship, and also as

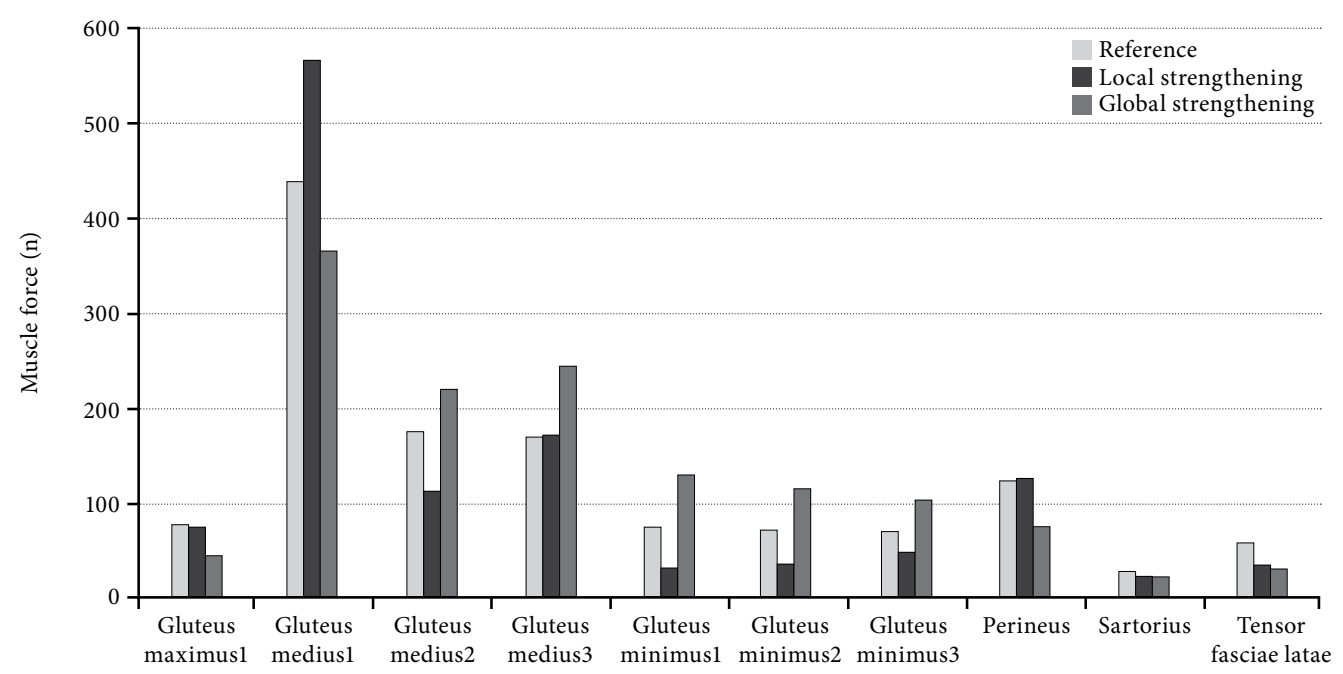

Figure 2. Maximum muscle forces during gait. Effect of strengthening of single muscle-tendon unit (glut med 1) and the whole musculus medius and minimus. 




Figure 3. Hip joint force during gait. Local strengthening of single muscle-tendon unit (glut med 1) compared to the effect of global strengthening of whole musculus medius and minimus.

important variables, such as muscle forces, are not often measurable. ${ }^{[6]}$

Strengthening of an individual muscle unit increases its load bearing considerably. Strengthened muscle becomes overloaded, while the activity of other abductors decreases compared to the reference state. Local muscle strengthening can be dangerous for hip stability, as the underloaded muscles may atrophy, while the overloaded muscle hypertrophy and hip disbalance may develop.

This study indicates that complex strengthening is more favorable. This statement, well-established empirically, has important biomechanical consequences. It has been shown that the global strengthening distributes force between more muscle and it dynamically stabilizes the hip. In addition, muscle fatigue is related to mechanical stress in muscles. ${ }^{[8]}$ Redistribution of the muscle forces between the muscles decreases the mechanical stress and, thus, improves the muscle performance.

Although the analysis was performed for a hip muscle, we consider that the described effect can be generalized. Muscles in the human body form a redundant system, i.e. there is always more muscles crossing a joint than needed to perform the given motion from mechanical point of view. ${ }^{[9]}$ Analysis was performed in a standard model, based on cadaver measurements and population averages of studied motion pattern.$^{[7]}$ However, inter-individual variations may influence the values and time-course of the force, although they would not likely change general conclusions of the study. Furthermore, the hypothesis of local strengthening could not be fully achieved in practice. Due to complex geometry of muscle in the human body, rehabilitation destined to one muscle segment influences synergic muscles, as well. However, the analysis has clearly identified the risk of single muscle hypertrophy.

In conclusion, the results of this study support adoption of complex training programs in rehabilitation practice. Based on biomechanical analyses, to achieve optimal performance of the musculoskeletal system, it is of utmost importance to include complex exercises which would improve not only the function of one muscle, but also improve the musculoskeletal system, as a whole.

\section{Declaration of conflicting interests}

The authors declared no conflicts of interest with respect to the authorship and/or publication of this article.

\section{Funding}

The study has been supported by the Medical Research Agency of the Czech Republic, project No. 15-33629A.

\section{REFERENCES}

1. Allen J, Butler S. The knee. In: Comfort P, Abrahamson E, editors. Sport Rehabilitation and Injury Prevention. Chippenham, Wiltshire: Wiley-Blackwell; 2010. p. 407-464.

2. Presswood L, Cronin J, Keogh J, Whatman C. Gluteus Medius: Applied Anatomy, Dysfunction, Assessment, and Progressive Strengthening. Strength and Conditioning Journal 2008;30:41-53.

3. Reiman MP, Bolgla LA, Loudon JK. A literature review of studies evaluating gluteus maximus and gluteus medius activation during rehabilitation exercises. Physiother Theory Pract 2012;28:257-68.

4. van der Krogt MM, Delp SL, Schwartz MH. How robust is human gait to muscle weakness? Gait Posture 2012;36:113-9.

5. Delp SL, Anderson FC, Arnold AS, Loan P, Habib A, John CT, et al. OpenSim: open-source software to create and analyze dynamic simulations of movement. IEEE Trans Biomed Eng 2007;54:1940-50.

6. Seth A, Sherman M, Reinbolt JA, Delp SL. OpenSim: a musculoskeletal modeling and simulation framework for in silico investigations and exchange. Procedia IUTAM 2011;2:212-232.

7. Delp SL, Loan JP, Hoy MG, Zajac FE, Topp EL, Rosen JM. An interactive graphics-based model of the lower extremity to study orthopaedic surgical procedures. IEEE Trans Biomed Eng 1990;37:757-67.

8. Crowninshield RD, Brand RA. A physiologically based criterion of muscle force prediction in locomotion. J Biomech 1981;14:793-801.

9. Collins JJ. The redundant nature of locomotor optimization laws. J Biomech 1995;28:251-67. 